\title{
Automated Indoor Air Quality Monitor and Control
}

\author{
D. Mahanth Mukesh \\ (Bachelor of Technology) \\ Department of Electronics and \\ Communication Engineering \\ GITAM University, Visakhapatnam \\ Andhra Pradesh, India
}

\author{
Sai Karishma Akula \\ (Bachelor of Technology) \\ Department of Computer Science \\ and Engineering \\ GITAM University, Visakhapatnam \\ Andhra Pradesh, India
}

\begin{abstract}
This paper presents a system of Automated Indoor Air quality Monitor which can be controlled and monitored using a mobile phone basing the concept of Internet of Things. The system monitors the indoor environment periodically and can be controlled using a Smart Phone. The system here is on internet of things platform which gives remote access through a mobile phone. The proposed system monitors the $\mathrm{CO}$ and $\mathrm{CO}_{2}$ levels in the room and notifies the user if the tolerable limit exceeds. The user can turn on the vent fan from the smart phone, when the tolerable limit exceeds. This system was implemented using raspberry pi, which uses a database to store the monitored values and a web server which can be accessed through the smart phone.
\end{abstract}

\section{Keywords}

Internet of things, Raspberry pi, NGINX, SQLite, Python, Relay, COZIR, MQ-7, Android Studio

\section{INTRODUCTION}

Carbon monoxide $(\mathrm{CO})$ and Carbon dioxide $\left(\mathrm{CO}_{2}\right)$ are odourless and colorless gases which show some adverse effects on humans if they exceed the tolerable level. These days $\mathrm{CO}$ and $\mathrm{CO}_{2}$ gases are higher indoors due to the increased human activities. $\mathrm{CO}_{2}$ is added to the indoor environments due to respiration and other activities. $\mathrm{CO}$ is added to the indoor environment due to unvented kerosene and gas space heater, leaking chimneys and furnaces, backdrafting from furnaces, gas water heaters, wood stoves and fireplaces, gas stoves, generators and other gasoline powered equipment, automobile exhaust from attached garages, tobacco smoke, etc. [1]. Higher $\mathrm{CO}_{2}$ levels affect the humans directly. A new study from the Harvard School of Public Health finds that carbon dioxide $\left(\mathrm{CO}_{2}\right)$ has a direct and negative impact on human cognition and decision-making. The Harvard study confirms the findings of a little-publicized 2012 Lawrence Berkeley National Laboratory (LBNL) study, "Is $\mathrm{CO}_{2}$ an Indoor Pollutant? Direct Effects of Low-toModerate $\mathrm{CO}_{2}$ Concentrations on Human Decision-Making Performance." That study found "statistically significant and meaningful reductions in decision-making performance" in test subjects as $\mathrm{CO}_{2}$ levels rose from a baseline of 600 parts per million (ppm) to $1000 \mathrm{ppm}$ and $2500 \mathrm{ppm}$ [2]. On the other hand Carbon monoxide ( $\mathrm{CO}$ ) affects the human health directly. At low concentrations health Effects Associated with Carbon Monoxide are fatigues in healthy people, chest pain in people with heart disease. At moderate concentration the effects are angina, impaired vision, and reduced brain function. At high concentrations the effects are impaired vision and coordination, headaches, dizziness, confusion, nausea, flu-like symptoms that clear up after leaving home, fatal at very high concentrations.
Indoor air quality monitoring is an essential thing which ensures that the indoor environment is safe for living. Indoor air quality monitoring system monitors the levels of the particular gases like $\mathrm{CO}_{2}, \mathrm{CO}, \mathrm{N}$, etc. This process of monitoring the air quality may be done using various methods. By monitoring the indoor environment, the levels of the respected gases which are harmful can be known. Even though these gasses cannot be avoided completely, they can be reduced to a tolerable and safe level for humans. This can be done by installing the Ventilation Fans and turning them $\mathrm{ON}$ whenever these $\mathrm{CO}$ and $\mathrm{CO}_{2}$ cross the safe levels [3].

In this paper the system proposed monitors the indoor air quality and also controls it. Here the $\mathrm{CO}$ and $\mathrm{CO}_{2}$ sensors are used to monitor their levels and ensure that they are in the base level. There are some related works on this topic [4][5][6][7][8], but this paper proposes the automation of the air quality monitor system through a Smart Phone using the Internet of things platform. With this system the indoor environment can be monitored and controlled remotely.

\section{SYSTEM ARCHITECTURE}

The system architecture of the proposed system for monitoring the indoor air quality is presented in Fig 1 . The architecture is based on the Internet of things platform where the proposed system is connected to the internet [9]. This system enables the user to remotely access the monitored data and control the ventilation fan.

Input Port: The General Purpose Input Output Ports receive the data from the sensors. These input ports act as an interface between the sensors and the raspberry pi. The data is now stored in the database installed in the raspberry pi. This data is processed and displayed to the user.

Web Server: The web server is an application which serves the web pages over HTTP. A web server is created on the raspberry pi which hosts the data base, enabling the user to access the data base remotely and communicate to the requests from the user while giving response to the requests.

Database: The data received from the sensors are stored in this database. The database is created on the raspberry pi web server. The database consists of data like time, level of $\mathrm{CO}$ and $\mathrm{CO}_{2}$, date. The data is received from the sensor in equal time frames.

Smart Phone: The smart phone consists of an android app which will be connected to the web server hosted by the raspberry pi. Whenever the threshold of the tolerable level is crossed, the app notifies the user with an alert that the air quality is low. Then the user gets two options: Turn ON and Turn OFF. When the ON button is pressed the request is sent to raspberry pi and it responds to the request by turning the relay circuit ON. When the relay is ON, the Fan Turns ON and the $\mathrm{CO}, \mathrm{Co}_{2}$ levels come down slowly. When the indoor 
level comes down the threshold level, the user again gets an alert that the air quality is good.

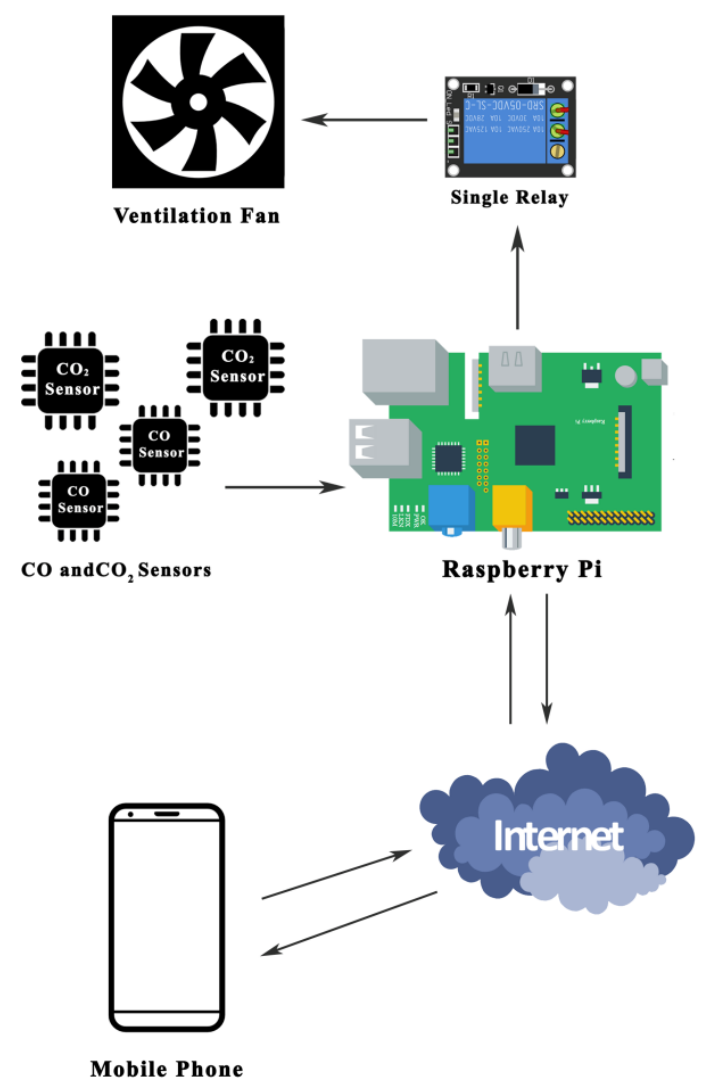

Fig 1: System Architecture

\section{HARDWARE DESIGN}

The Design of the System proposed is discussed in this section and type of sensors used.

\subsection{Carbon Monoxide Sensor MQ-7}

The Carbon Monoxide sensor MQ-7 is used here to detect the level of the CO gas in the indoor environment. It has good sensitivity to carbon monoxide in wide range, and has advantages such as long lifespan, low cost and simple drive circuit \& etc. Sensitive material of MQ-7 gas sensor is $\mathrm{SnO}_{2}$, which detects the $\mathrm{CO}$ gas. The target gas is Carbon monoxide and the detection range is 10 to $500 \mathrm{ppm}$.

The sensor requires two voltage inputs: heater voltage $\left(\mathrm{V}_{\mathrm{H}}\right)$ and circuit voltage $\left(\mathrm{V}_{\mathrm{C}}\right) . \mathrm{V}_{\mathrm{H}}$ is used to supply standard working temperature to the sensor and it can adopt DC or AC power. For this model sensor, $\mathrm{V}_{\mathrm{H}}$ should be at $1.5 \mathrm{~V} \pm 0.1 \mathrm{~V}$ at low temperature and $5 \mathrm{~V} \pm 0.1 \mathrm{~V}$ at high temperature. $\mathrm{V}_{\mathrm{RL}}$ is the voltage of load resistance RL which is in series with sensor. $\mathrm{V}_{\mathrm{C}}$ supplies the detect voltage to load resistance RL and it should adopt DC power [10].

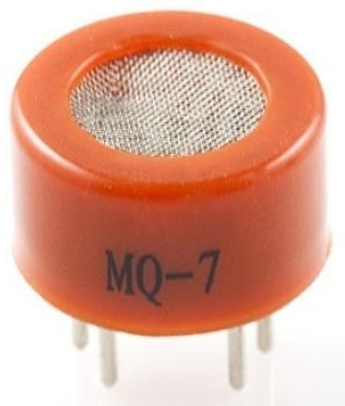

Fig 2: MQ-7 Sensor for Carbon Monoxide

Fig 2 shows the MQ-7 sensor manufactured by the Zhengzhou Winsen Electronics Technology Co., Ltd. The MQ-7 sensors are connected to the raspberry pi GPIO pins which act as the input pins. The data is processed and stored in the database.

\subsection{Carbon Dioxide Sensor COZIR-A}

The Carbon Dioxide sensor COZIR-A is used here to detect the level of $\mathrm{CO}_{2}$ in the indoor environment. COZIR is a $\mathrm{CO}_{2}$ module for OEM applications that represents a breakthrough in low power gas detection, making it ideal for battery powered, energy harvesting or wireless applications with tight energy budgets requiring long operating life. This Infrared LED based sensor offers a number of distinctive features over conventional $\mathrm{CO}_{2}$ sensors. Measurement ranges of $0-2000$ ppm, 0-5000 ppm and 0-1\% (10,000 ppm) [11].

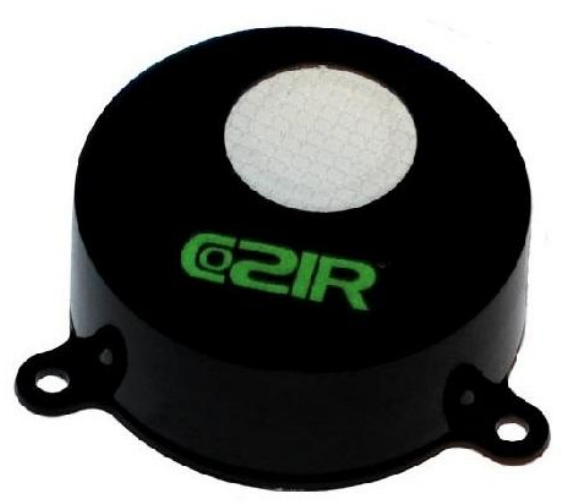

Fig 3: COZIR-A Sensor for Carbon Dioxide

Fig 3 shows the COZIR-A sensor manufactured by the AirTest Technologies Inc. The COZIR-A sensors are connected to the GPIO pins of the raspberry pi. The collected data from these sensors are processed and stored in the data base.

\subsection{Raspberry pi and Relay Circuit}

The raspberry pi is a credit card sized single board computer developed by the Raspberry Pi Foundation. The Raspberry Pi has an Ethernet port, alongside the USB ports. If Raspberry Pi is situated close to a router, access point, or switch, it can be connected to a network using an Ethernet cable. The latest version also has a built in WiFi. The Raspbian is a free operating system based on Debian, optimized for the Raspberry Pi hardware which can be installed using a Micro SD card. The GPIO pins on the board can be used as either input ports or output ports. The sensors are connected to these GPIO ports. The Raspbian OS is installed on the board which 
has Python, Scratch, Sonic Pi, Java, Mathematica and more [12]. Python Programming language can be used on the raspberry pi.

The Single Relay Board can be used to turn the ventilation fan on/off while keeping it isolated from the raspberry board. The Single Relay Board can control high-power devices (up to 10 A) via the on-board relay. This Relay board is connected to the raspberry pi GPIO pin which acts as an output pin [13].

\section{INTERNET OF THINGS PLATFORM}

The internet of things concept is used in this system to remotely monitor and control the system. In this section, the database, webserver and the android app used to access the system remotely are discussed [14] [15].

\subsection{SQLite Database}

SQLite is an embedded SQL database engine. Unlike most other SQL databases, SQLite does not have a separate server process. SQLite reads and writes directly to ordinary disk files. A complete SQL database with multiple tables, indices, triggers, and views, is contained in a single disk file. The database file format is a cross-platform - you can freely copy a database between 32-bit and 64-bit systems or between bigendian and little-endian architectures [16]. These features make SQLite a popular choice as an Application File Format. The database of the system is built on the raspberry pi using this SQLite and Python Programming Language. Two tables are inserted in which one is for the $\mathrm{CO}$ and other table for the $\mathrm{CO}_{2}$. The $\mathrm{CO}$ and $\mathrm{CO}_{2}$ tables consists the data of the date and time, sensor number, and the PPM of the gas. The PPM of the gas is monitored continuously by the sensor, but the PPM value is stored in the data base for every $\mathrm{n}$ minutes only. The data base can be accessed by the user anytime remotely in the smart phone by using the android app or by connecting to the server hosted by the raspberry pi.

Table 1. Sample of CO Database

\begin{tabular}{|c|c|}
\hline Time & PPM \\
\hline $19: 12: 37$ & 0 \\
$7-11-2016$ & 0 \\
\hline $19: 42: 37$ & 0 \\
$7-11-2016$ & 0 \\
\hline $20: 12: 37$ & \\
$7-11-2016$ & \\
\hline
\end{tabular}

Table 2. Sample of $\mathrm{CO}_{2}$ Database

\begin{tabular}{|c|c|}
\hline Time & PPM \\
\hline $19: 12: 37$ & 637 \\
$7-11-2016$ & 714 \\
\hline $19: 42: 37$ & 789 \\
$7-11-2016$ & $78: 12: 37$ \\
\hline $7-11-2016$ & \\
\hline
\end{tabular}

As shown above the data base is displayed whenever the user requests to view the monitored results.

\subsection{NGINX Webserver}

The webserver used in this system is NGINX which is a free, open-source, high-performance HTTP server. NGINX can serve HTML files over HTTP and with additional modules; it can serve dynamic web pages using scripting languages such as PHP [17]. The web content which can be accessed remotely by the user is hosted by this NGINX on the raspberry pi with flask frame work. The most commonly used
HTTP methods POST, GET, PUT, and DELETE can be used to perform actions on the web server. The webserver on the raspberry hosts the web content which displays the value of the $\mathrm{CO}$ and $\mathrm{CO}_{2}$ on the mobile. The data is refreshed continuously so that the indoor air quality is monitored continuously. The values monitored are just displayed in the app but not stored in the database. When a request is received by the user to display the monitored values then the values stored in the database are displayed. The webserver also receives request from the user to turn ON/OFF the fan and processes the request by turning the relay ON/FF.

\subsection{Android Application}

An android app is designed to access the system through the internet. The App on the smart phone is connected to the server hosted by the raspberry pi and receives the data monitored by the sensors. The data shown on the app is the PPM at that particular movement. The data monitored at particular intervals saved in the data base can also be accessed on request. The User Interface of the app is very simple. The app notifies an alert when the threshold PPM level is crossed. The user may switch ON/OFF the vent through the android app. The app contains two buttons ON and OFF to control the Fan. There is a separate tab to view the monitored data stored in the database. In that tab the average PPM value of the gases stored in the database at predetermined intervals of time are displayed.

\section{IMPLEMENTATION}

The first stage of proposed system is sensing the level of PPM of the gases in the indoor environment. For some accuracy, more than one sensor is placed in the room. In the second stage the sensor values obtained are processed to get the average value. The average value is compared with the threshold value in order to check whether the value is within the limit or not. If the value exceeds the threshold value then a notification is sent to the android app saying that the particular gas has crossed its threshold and it's not safe. If the average value is less than the threshold value then the system continues to monitor without any interruption. The sensor value is continuously monitored and the level of PPM is displayed on the app. But all the monitored values are not stored in the data base. The average value is stored into the database for every $\mathrm{n}$ minutes. This is done using the package index "schedule" which is a process scheduler for periodic jobs. Schedule lets us run python functions periodically at predetermined intervals [18]. According to the "American Society of Heating, Refrigerating and Air-Conditioning Engineers" they set the maximum levels of $\mathrm{CO}$ at $35 \mathrm{ppm}(1$ hour averaging) and $9 \mathrm{ppm}$ ( 8 hour averaging), while the World Health Organization limits $\mathrm{CO}$ concentrations based on exposure time ranging from $90 \mathrm{ppm}$ (15 minute exposure) to $10 \mathrm{ppm}$ (8 hour exposure) [19]. According to the LBNLSUNY study found a measurable negative impact on human cognition at $1000 \mathrm{ppm}$ of $\mathrm{CO}_{2}$. The Harvard researchers had a more comprehensive study that found significant negative impact at $930 \mathrm{ppm}$ of $\mathrm{CO}_{2}$. Clearly, more research needs to be done to know the threshold at which $\mathrm{CO} 2$ levels begin to show impact on human cognition. So taking this study into consideration we take the threshold for the Carbon Monoxide as 9 PPM and the threshold for $\mathrm{CO}_{2}$ as 1000 PPM. On average the $\mathrm{CO}$ level in an indoor environment is $0 \mathrm{PPM}$ and the $\mathrm{CO}_{2}$ level in an indoor environment varies from 400-800 PPM in normal conditions. The following Flowchart in Fig 4 shows how the data obtained from sensors is processed. 


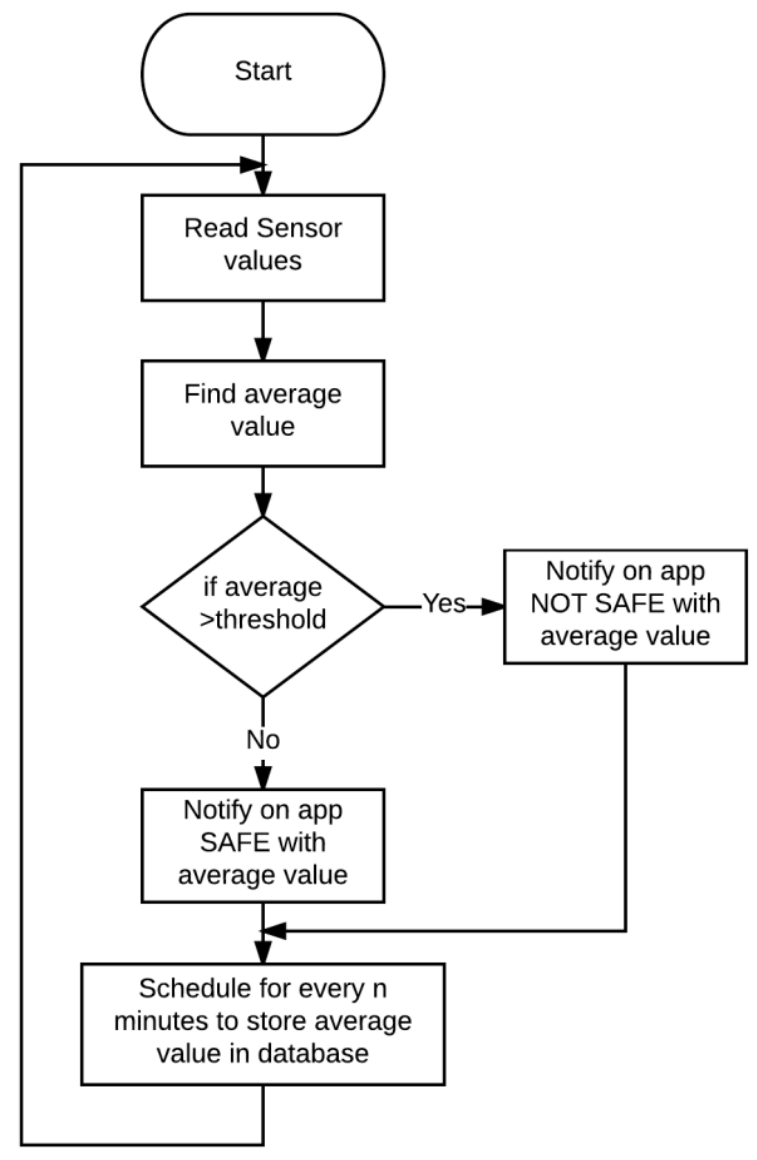

Fig 4: Flow chart for monitored values

The data processed in the raspberry pi is displayed on the android application through the web server. The third stage is displaying the data on the app. The system can be controlled by sending and receiving requests which are processed by the raspberry pi. The app is designed using the android studio and java programming language. The User interface of the app is easy to use and simple. The app connects to the web server through internet and accesses the information from raspberry pi. The app gets the current level of $\mathrm{CO}$ and $\mathrm{CO}_{2}$ along with the individual gas status, stating safe or not safe. The app displays the current level of $\mathrm{CO}$ and $\mathrm{CO}_{2}$ in green, if both the individual gas status is safe, and notifies the status of indoor environment as SAFE. If the status of any gas is not safe, then the app notifies the value of that particular gas in red, notifying the status of indoor environment as NOT SAFE. Values of both $\mathrm{CO}$ and $\mathrm{CO}_{2}$ gases are displayed with the status of the indoor environment below. The interface to control the Vent system is available below the indoor status. Two buttons ON and OFF are placed to turn ON/OFF the vent system. Status of the Vent system is placed below these buttons, in green if in ON state or in red if in OFF state. The history option is available in the menu of the app, through which the monitored values can be accessed by the user. The values are fetched from the database through internet. The data present in the history tab gives the information about time, date and PPM level of the gas, which is stored in database for every $\mathrm{n}$ minute's. The menu also has the exit option which can be clicked at any time to exit the app. The following Flowchart in Fig 5 shows how the app runs.

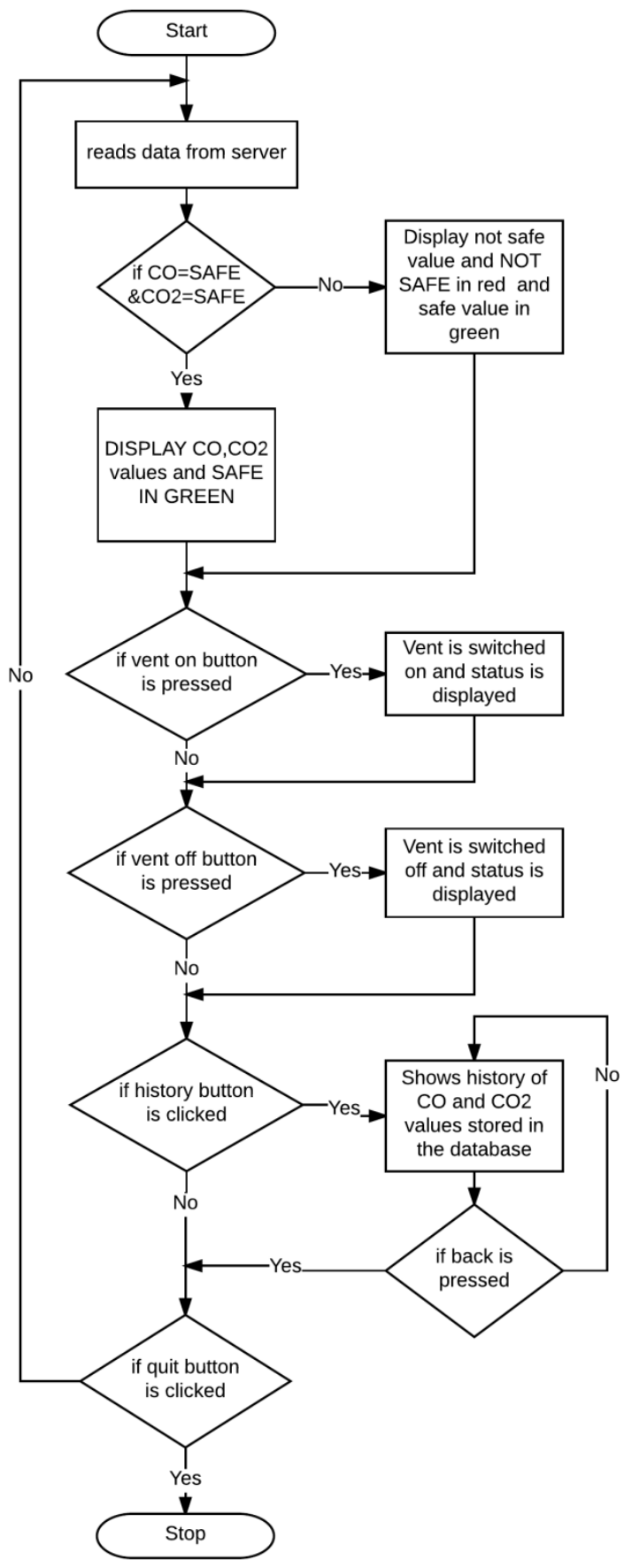

Fig 5: Flow chart for the app developed

\section{RESULTS AND DISCUSSION}

The experimental results obtained from the proposed system are shown below. The app is turned on and the monitored values of $\mathrm{CO}$ and $\mathrm{CO}_{2}$ level are displayed. The app menu has the history and exit options. In Fig 6 the CO value is 0 PPM, $\mathrm{CO}_{2}$ is $615 \mathrm{PPM}$ and the indoor environment status is SAFE. As the vent is in OFF state the status is displayed as "Vent System is OFF" in red. In Fig 7 the $\mathrm{CO}_{2}$ level crossed the threshold value and the status of $\mathrm{CO}_{2}$ is displayed in red and the indoor environment status as NOT SAFE. 

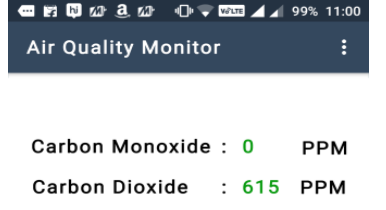

SAFE
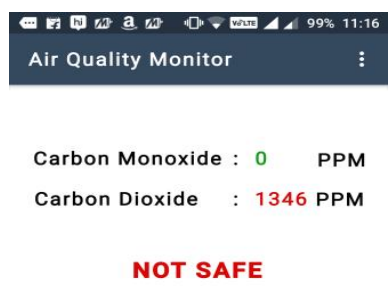

Vent Fan

Vent Fan
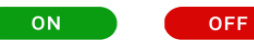

Vent System is OFF

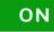

OFF

\section{ON}

Vent System is OFF
Fig 6: SAFE status

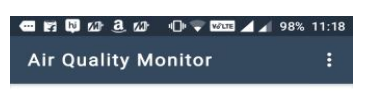

Carbon Monoxide : $0 \quad$ PPM

Carbon Dioxide : 1362 PPM

NOT SAFE

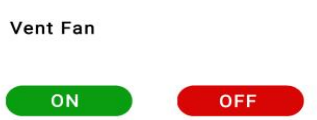

Vent System is ON
Fig 7: NOT SAFE status

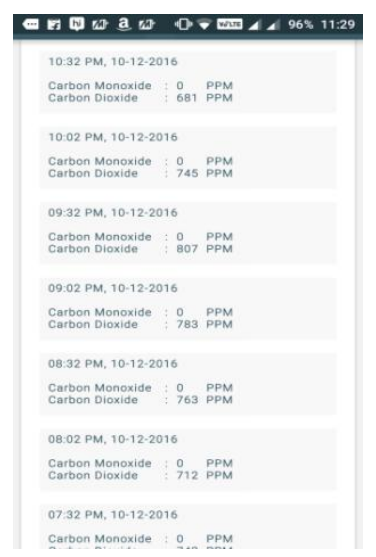

Fig 9: Monitored data
Fig 8: Vent in turned $O N$

In Fig 8 the Vent system is turned $\mathrm{ON}$ and the status of vent system is displayed as "Vent System is ON" in green. In Fig 9 the User interface of the history tab is shown, which contains the data of monitored values of PPM at equal intervals of time.

\section{CONCLUSION}

The development of the proposed system Indoor Air Quality Monitor and Control, which monitors the Air quality by measuring the $\mathrm{CO}, \mathrm{CO}_{2}$ levels and gives user remote access to the monitored data and the Vent system is presented. This system can be used to monitor the indoor environment in class rooms, waiting rooms, office, and other crowded places. This system is based on the Internet of things platform which adds the feature to control the vent system remotely, which is useful in maintaining the indoor environments without suffocation. This system provides the user an ease to control the indoor environment up to an extent which reduces the adverse effects due to high level of gases like $\mathrm{CO}$ and $\mathrm{CO}_{2}$.

\section{FUTURE SCOPE}

Further this system can be enhanced with some added extra features like detection of other harmful gases and oxygen level indicator. Further work intends to increase the reliability and accuracy of the system. The system may be modified to monitor the indoor environment in different rooms of a building and display the individual data of each room.

\section{ACKNOWLEDGMENTS}

The authors would like to acknowledge the work of Lawrence Berkeley National Laboratory on Carbon Dioxide and
American Society of Heating, Refrigerating and AirConditioning Engineers for their work on "Ventilation for Acceptable Indoor Air Quality". The authors would like to thank U. Rahul Varma for his valuable help.

\section{REFERENCES}

[1] Carbon Monoxide's Impact on Indoor Air Quality. [Online]. Available: https://www.epa.gov/indoor-airquality-iaq/carbon-monoxides-impact-indoor-air-quality

[2] CO2 Levels Directly Affect Human Cognition, New Harvard Study Shows. [Online]. Available: https://thinkprogress.org/exclusive-elevated-co2-levelsdirectly-affect-human-cognition-new-harvard-studyshows-2748e7378941\#.r04njqq7c

[3] Preethichandra, D. M. G. "Design of a smart indoor air quality monitoring wireless sensor network for assisted living." 2013 IEEE International Instrumentation and Measurement Technology Conference (I2MTC). IEEE, 2013.

[4] Li, Jiaming, Josh Wall, and Glenn Platt. "Indoor air quality control of HVAC system." Modelling, Identification and Control (ICMIC), The 2010 International Conference on. IEEE, 2010.

[5] Han, Zhenyu, Robert X. Gao, and Zhaoyan Fan. "Occupancy and indoor environment quality sensing for smart buildings." Instrumentation and Measurement Technology Conference (I2MTC), 2012 IEEE International. IEEE, 2012.

[6] Lohani, Divya, and Debopam Acharya. "SmartVent: A Context Aware IoT System to Measure Indoor Air Quality and Ventilation Rate." Mobile Data Management (MDM), 2016 17th IEEE International Conference on. Vol. 2. IEEE, 2016.

[7] Kelly, Sean Dieter Tebje, Nagender Kumar Suryadevara, and Subhas Chandra Mukhopadhyay. "Towards the implementation of IoT for environmental condition monitoring in homes." IEEE Sensors Journal 13.10 (2013): 3846-3853.

[8] Srivatsa, Prajwala, and Amit Pandhare. "Indoor Air Quality: IoT Solution." National Conference "NCPCI. Vol. 2016. 2016.

[9] Mois, George, Teodora Sanislav, and Silviu C. Folea. "A Cyber-Physical System for Environmental Monitoring." IEEE Transactions on Instrumentation and Measurement 65.6 (2016): 1463-1471.

[10] MQ-7 Data Sheet- Zhengzhou Winsen Electronics Technology Co., Ltd

[11] COZIR-A Data Sheet- AirTest ${ }^{\mathrm{TM}}$ Technologies Inc.

[12] RASPBERRY PI DOCUMENTATION. [Online] Available: https://www.raspberrypi.org/documentation/

[13] Single Relay Board Documentation [online] Available: https://www.parallax.com/sites/default/files/downloads/2 7115-Single-Relay-Board-Guide-v1.0.pdf

[14] Zanella, Andrea, et al. "Internet of things for smart cities." IEEE Internet of Things Journal 1.1 (2014): 2232. 
[15] Valerio, Pablo. "Is the IoT a Tech Bubble for Cities?: With more cities joining the smart city revolution and investing in sensors and other IoT devices, the risk of a new tech bubble is rising." IEEE Consumer Electronics Magazine 5.1 (2016): 61-62.

[16] SQLite. [Online]. Available: http://www.sqlite.org
[17] RASPBERRY PI DOCUMENTATION. [Online] Available: https://www.raspberrypi.org/documentation/

[18] Python Documentation [Online] Available : https://www.python.org/doc/

[19] Janssen, John E. "Ventilation for acceptable indoor air quality." ASHRAE journal 31.10 (1989): 40-48. 